\title{
Verbale Konstruktionen auf dem Weg wohin? peut-être, maybe, scheints
}

\section{Einleitung}

In Beispielen wie

(1) Du hast scheints / Weiß Gott nichts begriffen.

(2) It cost $£ 200$, give or take.

(3) Qu'est ce qu'il a dit?

werden verbale Konstruktionen (kurz: VK, hier jeweils die fett gesetzten Teile) in einer Weise gebraucht, die der Grammatik verbaler Konstruktionen zuwiderläuft. In (1) und (2) wird die verbale Konstruktion wie ein Adverb/eine Partikel gebraucht bzw. wie ein Ausdruck in der Funktion eines (adverbialen) Adjunkts/ Supplements. In (3) ist die verbale Konstruktion zum Bestandteil einer periphrastischen interrogativen Konstruktion geworden. Wie sind solche 'Umfunktionalisierungen' - wie ich das Phänomen zunächst vortheoretisch bezeichnen möchte - einzuordnen? Handelt es sich um Lexikalisierung oder um Grammatikalisierung? Oder um ein Phänomen der dritten Art? Die Umfunktionalisierung verbaler Syntagmen bzw. Konstruktionen - ich gebrauche die Abkürzung UVK für 'umfunktionalisierte verbale Konstruktion(en)' - ist ein bisher weniger gut untersuchtes Phänomen, etwa gegenüber der Umfunktionalisierung von Präpositionalphrasen, die sprachübergreifend zu komplexen, „sekundären“ Präpositionen werden können (man vergleiche DEU auf Grund + Genitiv / von, ENG on top of, FRA à cause de). Eine Ausnahme bilden hier verbale Konstruktionen, die zu Tempus- und Aspekt-Markern geworden sind wie etwa in der synthetischen Futur-Konstruktion der romanischen Sprache (wie FRA chantera > LAT cantare habet; vgl. Lehmann 1985: 308) oder auch analytische Konstruktionen wie die englische Futur-Konstruktion mit to be going to bzw. die französische aller + Infinitiv-Konstruktion oder auch die in verschiedenen europäischen Sprachen belegten Perfekt-Konstruktionen mit haben/have/avoir/avere usw. + Partizip Perfekt (Lehmann 2015: 31-34). In diesen Fällen handelt es sich eindeutig um Grammatikalisierung, wenn man sich an der Definition von Meillet (1948 [1912]: 131) als ,attribution du caractère grammatical à un mot jadis autonome“ orientiert. Sie wurden verschiedentlich geradezu als exemplarische Fälle von Gram- 
matikalisierung eingeordnet und intensiv untersucht. Auf der anderen Seite wurden verbale Kollokationen genauer analysiert, die den Status von Idiomen, Phrasemen oder Phraseologismen haben, wie etwa jemandem einen Bären aufbinden, jemandem ein $X$ für ein $U$ vormachen. Hier ist die Bedeutung nicht mehr wörtlich zu nehmen oder kompositional herzuleiten; die grammatische Kategorie des Ausdrucks (als Verbalphrase) und ihre syntaktische Funktion (als teilweise durch Komplemente gesättigtes Prädikat) jedoch bleibt unberührt. Die Umfunktionalisierungen der mit (1) bis (3) genannten Art sind demgegenüber weniger klar umrissen. In dem vorliegenden Aufsatz soll daher der Versuch unternommen werden, den systematischen Status von UVK, die nicht zu grammatischen Markern oder Idiomen (im erläuterten Sinne) geworden sind, zu klären. Dabei werden verbale Konstruktionen in erster Linie aus dem Deutschen, aber auch aus dem Englischen und Französischen, in den Blick genommen. Anzumerken ist hierzu, dass die Phänomene im Einzelnen durchaus grammatikografisch und ggf. lexikografisch erfasst werden, eine Systematisierung unter dem Gesichtspunkt des so gegebenen Potenzials zur Umnutzung verbaler Konstruktionen - jenseits ihrer zentralen Funktion als Satzprädikat - steht jedoch noch aus. Vermehrtes Interesse findet neuerdings sprachübergreifend die Umfunktionalisierung verbaler Konstruktionen zu Diskursmarkern; vgl. für das Deutsche etwa Auer/Günthner (2005), für das Französische Dostie (2013). Dieser Gebrauch wird hier nicht thematisiert, man vergleiche jedoch Fußnote 8. Eine zweite Zielsetzung des Beitrags besteht somit auch darin, sprachübergreifende und sprachspezifische Eigenschaften solcher Prozesse der Umfunktionalisierung zumindest im Ansatz herauszuarbeiten.

\section{2 Überblick zu den verschiedenen Konstruktionstypen}

Bei der folgenden Übersicht werden die Daten nach zwei Gesichtspunkten geordnet: a) nach der zugrundeliegenden verbalen Konstruktion und b) nach der Zielkategorie und der Zielfunktion, also der Kategorie und Funktion nach der Umfunktionalisierung. Bei Gesichtspunkt a) ist also zum einen danach gefragt, um welche Verbform es sich handelt, bei finiten Formen ist die Person-, Numerus- und Tempuskategorie gefragt sowie auch der Verbmodus (Indikativ/Konjunktiv), bei infiniten die Einordung als Infinitiv, Partizip oder Imperativ. Zu letzterem ist jedoch gleich eine Einschränkung zu machen: Infinitivische Konstruktionen werden häufig als selbstständige Äußerungseinheiten, meist mit direktivem Illokutionstyp gebraucht, wie etwa bei wie Den Rasen nicht betreten, 
Alle mal herhören. Diese Fälle beziehe ich nicht ein, ebenso wenig den Gebrauch von Perfektpartizipien bzw. entsprechenden Partizipialphrasen als selbstständige Äußerungseinheiten meist ebenfalls direktiver Natur (wie Aufgepasst!). Abgesehen wird auch von der Konversion von Partizipien (als Verbformen) in Adjektive, da es sich hier um einen gut erforschten, äußerst produktiven Prozess handelt, bei dem möglicherweise gar nicht von Umfunktionalisierung bzw. Konversion, sondern von einer per se doppelten Kategorienzugehörigkeit gesprochen werden sollte. Im Vordergrund stehen somit finite Verbformen und Imperative als verbaler Kern des Konstruktionstyps. Partizipiale Konstruktionen werden nur einbezogen, wenn bei ihnen ein Kategorienwechsel ungleich dem Wechsel zu der Kategorie Adjektiv oder selbstständige Äußerungseinheit stattgefunden hat. Als zweiter Aspekt neben der Kategorie der Verbform ist die Art ihres phrasalen Ausbaus innerhalb der Konstruktion zu berücksichtigen. Hier offenbart sich besonders der Unterschied zu Umfunktionalisierungen, bei denen die Kernkonstituente ein Substantiv ist - sei es beim Ausbau zu einer NP (wie in letzten Endes, meines Erachtens) oder vor allem zu PPs (wie in Folge + Genitiv / von, in Bezug $a u f)$. Anders als in diesen Fällen liegt bei verbalen Konstruktionen eine große Vielfalt an phrasalen Ausbaumöglichkeiten vor, die auf das spezifische und konstruktionsabhängig variable Valenzpotenzial des Verbs zurückgehen. Während Substantive keine oder maximal eine Leerstelle eröffnen, und bei Einbindung in eine PP, die auf dem Wege der Grammatikalisierung zur Präposition ist, stereotyp eine Leerstelle eröffnet wird, können Verben als bereits bezüglich ihrer Leerstelle(n) abgesättigte Ausdrücke umfunktionalisiert werden (wie in Gott sei Dank) oder aber über eine offene Leerstelle mit ihrer syntaktischen Umgebung verknüpft sein (wie in Gott weiß wer/wo/wie usw.). Aber auch wo nicht explizit Leerstellen eröffnet werden, ist die UVK in der Regel durch eine implizite Leerstelle mit dem Satzkontext verknüpft. Die Proposition des umgebenden Satzes ist ursprünglich als Argument der VK zu verstehen. Dies kann exemplarisch an ENG maybe und FRA peut-être gezeigt werden.

Ausgangskonstruktion

ENG It may be (that) they have misunderstood that. FRA II peut être qu'ils ont mécompris ça.

\section{Zielkonstruktion}

ENG They have maybe misunderstood that. FRA lls ont peut-être mécompris ça.

In beiden Sprachen geht die UVK auf eine Konstruktion zurück, in der die Verbindung aus einer Modalverbform (may, peut) und Kopulaverb (be, être) einen abhängigen Satz regiert, der den möglichen Sachverhalt beschreibt. In der Zielkonstruktion ist der abhängige Satz zum Hauptsatz „promoviert“ worden, als 
dessen Satzglied nun die UVK erscheint. Wir haben es also mit einer Dependenzumkehrung (vgl. Gunkel et al. 2017: 1627f.) zu tun.

Verbunden mit dieser Restrukturierung ist, dass die ursprüngliche Subjektstelle in einer Art Pro-Drop bzw. Topik-Drop entfallen muss; im eben genannten Fall entfällt somit das formale Subjekt ENG it, FRA il. wie in maybe > it may be (that)). Auch die deutsche Entsprechung es kann sein, (dass) erscheint in informaler Sprache in der Pro-Drop-Variante kann sein. Aber im Deutschen ist die Weglassung des nicht-phorischen es nicht zugleich mit einer vollständigen Umfunktionalisierung zur Modalpartikel verbunden. Vielmehr bleibt die Konstruktion aus VK + abhängigem Satz im Prinzip erhalten.

Pro-Drop ist allerdings nicht auf das nicht-phorische 'es' beschränkt; auch die Pronomina der 1. Person können entfallen, was besonders charakteristisch ist für UVK auf der Basis der performativen oder einstellungsbekundenden Verwendung von Verba dicendi oder sentiendi (wie in DEU schätze mal) oder in dem prominenten Fall der sprachübergreifenden Umfunktionalisierung von '(ich) bitte' zum illokutionsbezogenen Marker der höflichen Bitte. ${ }^{1}$

Hinzu kommen nicht valenzbedingte Erweiterungen durch Vergleichspartikeln (wie folgt, wie gehabt) oder durch adverbiale Bestimmungen (gelinde gesagt). Diese Vielfalt oder auch mangelnde Systematik des phrasalen Ausbaus mag einer der Gründe sein, warum es anders als bei PP nicht zu Reihenbildungen (aufgrund von Analogie) oder gar zu einer klaren Musterbildung gekommen ist.

Was den Aspekt b) angeht, die Zuordnung einer Zielkategorie und/oder einer Zielfunktion, so verstehe ich Kategorie hier zunächst im engeren Sinne der Zuordnung einer Wortart, wobei ich die Wortarten-Klassifikation der IDS-Grammatik (1997) zugrunde lege. Im Wesentlichen ist hier die Kategorie Partikel, insbesondere der Klassen Konnektivpartikel (wie erstens, übrigens) und Modalpartikel (wie vielleicht, leider) einschlägig; ggf. auch, siehe die Diskussion in Abschnitt 3.4, die Klasse Intensitätspartikel (wie sehr, ziemlich). ${ }^{2}$ Ich ordne den umfunktio-

1 So etwa neben dem Deutschen auch im älteren Englisch bei pray sowie im Lateinischen bei quaeso oder im Italienischen bei prego. Man vergleiche zur Entwicklung in den romanischen Sprachen sowie insgesamt zu verbgestützten „pragmatischen Markern“ den Sammelband von Ghezzi/Molinelli (Hgg.) (2014).

2 Zu beachten ist bezüglich des Terminus 'Modalpartikel', dass es sich nach dem Verständnis der IDS-Grammatik um eine Partikelklasse handelt, deren Elemente semantisch auf den Geltungsanspruch bzw. den Faktizitätsgrad der Proposition bezogen sind und die sowohl im Vorfeld als auch im Mittelfeld erscheinen können. Häufig wird allerdings der Terminus auch auf die Partikelklasse bezogen, die in der IDS-Grammatik 'Abtönungspartikeln' heißt und deren Elemente gerade nicht im Vorfeld erscheinen können. Zu einer Übersicht über die Partikelklassifikation der IDS-Grammatik vgl. auch Diewald (2013: 28). 
nalisierten verbalen Konstruktionen allerdings nur dann eine Wortart $\mathrm{zu}$, wenn sie sich lexikalisch und syntaktisch vollständig wie die „geborenen“ Elemente dieser Klassen verhalten, also als 'komplexe Lexeme' oder 'Phraseme'3 sowohl lexikalisch stabil und unveränderlich sind als auch dieselbe syntaktische Distribution wie lexikalisch einfache Elemente der Wortklasse haben und sowohl syntaktisch als auch vor allem prosodisch in den einbettenden Satz integriert sind. Häufig ist dies jedoch aufgrund des unvollständigen Grades der Umfunktionalisierung nicht der Fall. Hier kann es sich um variable Syntagmen handeln (wie etwa ehrlich/gelinde/offen gesagt) oder um Phraseme, die keine wortartanaloge Distribution haben - wie etwa ENG how come, das funktional dem Frageadverb why entspricht, aber sich syntaktisch anders verhält - oder die nur prosodisch desintegriert bzw. parenthetisch vorkommen - wie etwa FRA coûte que coûte. In diesen Fällen gehe ich nur von einer Funktionsähnlichkeit mit einer entsprechenden Wortart aus (im Zeichen ' '). Neben der Zuweisung einer Wortart ist die syntaktische und semantische Funktion, die die umfunktionalisierte verbale Konstruktion hat, zu bestimmen. Auch hier schließe ich mit gewissen Modifikationen an die IDS-Grammatik an. Als syntaktische Funktion, die hier in Frage kommt, identifiziere ich in erster Linie die Supplementation/Supplementfunktion, also die syntaktische Funktion nicht-valenzgebundener „primärer Komponenten“. Dabei lege ich wie in der IDS-Grammatik einen weiten Supplement-Begriff zugrunde, bei dem die Unterschiede zwischen adverbialen Angaben im engeren Sinne, die sich in jeder Hinsicht wie Satzglieder verhalten, und „Kommentargliedern“, die nur einen eingeschränkten Satzgliedstatus haben und die ggf. präferiert nur desintegriert vorkommen, nicht berücksichtigt werden. Daneben wird ein weiterer Aspekt herangezogen: die semantische Funktion als Modifikator der Proposition oder des mit der Proposition verbundenen Geltungsanspruchs. Ausdrücke mit letzterer Funktion, die auch als „modale“ Modifikatoren bezeichnet werden können, spielen hier die wichtigste Rolle. Sie sind in weitere Subklassen zu differenzieren: evidenzbetonend-assertive Modifikatoren (wie offensichtlich, zweifelsohne), bewertend-assertive (wie leider, unglücklicherweise) und modalabschwächende (wie möglicherweise, wahrscheinlich). Ebenfalls zu berücksichtigen sind geltungsneutrale Erweiterungen, die diskursorganisatorische oder metakommunikative Funktion haben. Daneben ist die Attribution einzubezie-

3 Von 'komplexem Lexem' spreche ich, wenn UVK vollständig univerbiert ist. Der Terminus 'Phrasem' bezeichnet hier feste und invariable Einheiten mit erkennbarer - in der Regel auch orthografisch erfasster - morphosyntaktischer Binnenstruktur, die also aus mehreren Wortformen bestehen und die man auch als Mehrwortlexeme oder Wortgruppenlexeme bezeichnen könnte. 
hen, hier zu verstehen als Ausbau beliebiger Phrasen über ihren Kernbestand hinaus, man denke etwa an den attributiven Ausbau von NPs, aber auch die Erweiterung von Adjektiven durch adadjektivisch gebrauchte Intensitätspartikeln (wie in Das ist [sehr schön] /eine [sehr schöne] Sache).

Wortarten und syntaktisch-semantische Funktionen stehen bekanntlich in der Regel in enger Korrelation. Im vorliegenden Fall etwa werden Ausdrücke, denen wir die Zielkategorie der Modalpartikel zuordnen wie ENG maybe, FRA peut-être, in der syntaktischen Funktion des Supplements gebraucht und haben die semantische Funktion eines modal-abschwächenden Modifikators. Die Zuweisung einer Zielwortart impliziert somit die Zuweisung einer syntaktisch-semantischen Funktion, während die umgekehrte Implikationsrichtung nicht gilt.

Schließlich gibt es Umfunktionalisierungen, die weder zu neuen Elementen einer der gängigen Wortarten führen noch zu einem komplexen Ausdruck mit eigenständiger syntaktischer und semantischer Funktion, sondern die als Bestandteile einer periphrastischen Konstruktion zu betrachten sind, der nur insgesamt eine solche Funktion zugeordnet werden kann. Ein Beispiel ist hier die eingangs genannte periphrastische Interrogativkonstruktion des Französischen.

Ich gebe nun einen tabellarischen Überblick zu umfunktionalisierten verbalen Konstruktionstypen nach Maßgabe der soeben eingeführten Parameter. Daran anschließen wird sich eine detailliertere Diskussion, auch anhand von Belegen:

Tab. 1: verbale Ausgangskonstruktion

\begin{tabular}{ll}
\hline Parameter & Kategorien \\
\hline Verbform & Imperativ: DEU Gott sei Dank, sage und schreibe, ENG give or take \\
& Partizip Perfekt: DEU wie gehabt/gelinde gesagt \\
& 1SG Präsens Indikativ: schätze (mal) \\
& 3SG Präsens Indikativ: DEU Weiß Gott, Gott weiß +w-Wort, wie folgt, ENG \\
& God/Heaven knows + wh-Wort, maybe, FRA peut-être, n'importe + qu-Wort \\
& 3SG Präsens Konjunktiv/subjonctif: DEU Gott bewahre, komme, was (da) \\
& wolle, FRA coûte que coûte, vaille que vaille, (vienne que vienne), qu-Wort \\
& + que ce soit \\
\hline interner phrasaler & Verbformen (koordiniert): z.B. sage und schreibe \\
Ausbau & Verbform + Komplement(e): z.B. Gott sei Dank \\
& Verbform + Supplement: gelinde gesagt \\
& Verbform + Vergleichspartikel: z.B. wie gesagt \\
\hline Gesättigtheit & gesättigt: z.B. Gott sei Dank, wie gehabt/gesagt \\
& + explizite Leerstelle: z.B. Gott/wer weiß+ w-Wort \\
Topik-Drop & Il peut être > peut-être \\
\hline
\end{tabular}


Tab. 2: Zielwortart/syntaktische und semantische Funktion der Zielkonstruktion

\begin{tabular}{lll}
\hline $\begin{array}{l}\text { Kategorialer Status/ } \\
\text { syntaktische Distribution } \\
\text { und Integration }\end{array}$ & Wortart & $\begin{array}{l}\text { syntaktische/semantische Funktion der } \\
\text { Zielkonstruktion }\end{array}$ \\
\hline
\end{tabular}

Komplexes Wort/Phrasem, Modalpartikel Supplement/Modifikation des Geltungswortartanaloge Distribution, integriert

anspruchs

evidenzbetonend-assertiv: DEU Weiß Gott bewertend-assertiv: DEU gottseidank modal-abschwächend: ENG maybe, FRA peut-être, DEU (Umgangssprache, Dialekte) scheints

$\begin{array}{ll}\text { (variables) Syntagma, } & \sim \text { Konnektiv- } \\ \text { wortartanaloge Distribution, } & \text { partikel } \\ \text { prosodisch meist desinte- } & \\ \text { griert } & \end{array}$

Supplement/diskursorganisatorische/ metakommunikative Modifikation wie folgt, anders/besser/genauer/... gesagt/formuliert/...

\begin{tabular}{|c|c|c|}
\hline $\begin{array}{l}\text { Phrasem, } \\
\text { keine wortartanaloge } \\
\text { Distribution }\end{array}$ & $\sim$ Adverb & $\begin{array}{l}\text { Supplement/prädikats- oder propositions- } \\
\text { bezogene Modifikation } \\
\text { ENG how come } \\
\text { DEU koste es, was es wolle, FRA coûte que } \\
\text { coûte }\end{array}$ \\
\hline $\begin{array}{l}\text { Phrasem, } \\
\text { keine wortartanaloge } \\
\text { Distribution }\end{array}$ & $\begin{array}{l}\sim \text { Intensitäts- } \\
\text { partikel }\end{array}$ & $\begin{array}{l}\text { Supplement/Attribut/Modifikation einer } \\
\text { Quantifikation } \\
\text { DEU sage und schreibe, ENG give or take }\end{array}$ \\
\hline Teil einer Periphrase & & $\begin{array}{l}\text { Teil einer periphrastischen Interrogativ- } \\
\text { konstruktion } \\
\text { FRA Qui/Qu'/Quand/... est ce qui/que, ... }\end{array}$ \\
\hline & & $\begin{array}{l}\text { Teil einer periphrastischen Adverbialkonstruk- } \\
\text { tion } \\
\text { ENG That's how/why/when/... } \\
\text { Teil einer periphrastischen Indefinitkonstruk- } \\
\text { tion } \\
\text { DEU Gott/wer weiß wer/was/wo/wann/... } \\
\text { ENG God/Heaven knows who/what/where/ } \\
\text { when, ... } \\
\text { FRA n'importe qui/que/où,..., } \\
\text { qui/que/où ... que ce soit }\end{array}$ \\
\hline
\end{tabular}




\section{Zu den Konstruktionstypen im Einzelnen}

\subsection{Umfunktionalisierung eines komplexen Lexems/Phrasems zur Modalpartikel}

Dies ist ein in allen drei Sprachen gängiger, vielleicht der gängigste Typ von UVK. Dabei hat sich kein einheitliches Strukturmuster etabliert, aber es sind doch strukturelle Generalisierungen erkennbar. Zwar sind unterschiedliche Verbformen vertreten (Imperativ, 3SG Indikativ/Konjunktiv), es kann Pro-Drop zugrunde liegen (wie bei maybe, peut-être) oder wie bei DEU scheints das nicht-phorische Pronomen es an die Verbform unter Vokalelision enklitisch angefügt erscheinen. Der spezifische semantische Charakter, also die Betonung der Evidenz, die Bewertung oder Abschwächung des Geltungsanspruchs wird auf zwei verschiedene Weisen zum Ausdruck gebracht: Durch Berufung auf eine höhere Instanz (Gott), oder durch die lexikalische Semantik eines Modal- oder Halbmodalverbs. Gemeinsam ist den vorliegenden UVK aber eindeutig die oben geschilderte dependenzumkehrende Reanalyse.

Der folgende Beleg zu DEU scheints illustriert den hohen Grad der Umfunktionalisierung zu einer Modalpartikel: scheints ist hier syntaktisch und prosodisch voll integriertes Supplement im Mittelfeld des Satzes.

(4) Und es war scheints alles so einfach: Die Utensilien für den Bau einer Rohrbombe sind im Handel ohne Probleme erhältlich, [...] (Niederösterreichische Nachrichten, 22.10.2007: 5)

Die Modalpartikel scheints ist in den süddeutschen Dialekten, aber wie DEREKoBelege zeigen, durchaus auch in der regionalen Pressesprache, vertreten, z.B. in Schweizer und österreichischen Zeitungen oder den „Nürnberger Nachrichten“.

ENG maybe und FRA peut-être sind als komplexe Lexeme zu betrachten, während DEU Gott sei Dank/gottseidank, zumindest wenn man nach der Schreibung geht, zwischen dem Status als Phrasem und dem als komplexes Wort schwankt. Das wörtliche deutsche Äquivalent $\mathrm{zu}$ den beiden genannten modal-abschwächenden Partikeln des Englischen und Französischen, nämlich kann sein, ist nicht zur Modalpartikel umfunktionalisiert; allenfalls eine erste Stufe ist in Form von Pro-Drop verwirklicht, während die zugrunde liegende syntaktische Struktur mit abhängigem dass-Satz oder abhängigem Verbzweitsatz erhalten bleibt:

(5) Ich werde vermutlich Schmerzen haben, werde desorientiert sein; kann sein, ich bin lange krank. Kann sein, ich werde Hilfe brauchen beim 
Sterben, jemanden, der mich mit Opiaten versorgt, [...] (Die Presse, 17.8.2014: 34)

Nur syntaktisch desintegriert, als Parenthese oder eigene kommunikative Minimaleinheit wird der Konstruktionstyp < Verbform 1SG Präsens Indikativ, ProDrop> wie DEU schätze, meist in der Verbindung schätze mal gebraucht. Hier liegt der Gebrauch eines Kognitionsverbs zum Zweck einer Modalisierung vor.

(6) Nach den Bauschildern soll die Brücke Anfang 2011 fertig sein. Dies wird definitiv nicht der Fall sein, schätze mal mindestens ein Jahr später. (http://de.wikipedia.org/wiki/Diskussion:Unstruttalbrücke: Wikipedia, 2011)

In diesem Kontext bezieht sich die Modalisierung auf den Zeitpunkt der Fertigstellung einer Brücke, also einen Sachverhaltsaspekt. Es liegt somit ein enger propositionsbezogener Skopus vor. Verben des Denkens werden, so heißt es in der Literatur (vgl. Fußnote 8), bevorzugt auch umfunktionalisiert zu Diskursmarkern. Dann liegt ein erweiterter Skopus vor. Ob und inwiefern diese Art der ,pragmatischen“ Umfunktionalisierung mit den hier betrachteten Typen Gemeinsamkeiten aufweist, wird aus Platzgründen nicht angesprochen.

\subsection{Umfunktionalisierung eines variablen Syntagmas zum metakommunikativen oder diskursorganisatorischen Modifikator}

Syntagmen der Art <adverbiales Supplement + Partizip Perfekt eines Verbums dicendi> stellen im Deutschen ein variables Strukturmuster metakommunikativer „Diktumserweiterungen“ dar; man vergleiche etwa besser/ehrlich/kurz/... gesagt, genauer formuliert, anders ausgedrückt, im Klartext gesprochen usw. In HDK2 (2014: 1130-1167) werden diese und ähnliche Syntagmen als „metakommunikative Konnektoren“ der Subklasse nicht nacherstfähige Adverbkonnektoren eingeordnet. ${ }^{4}$ Bereits in HDK1 (2003: 336) werden nicht nur wortförmige und phraseologische Einheiten, sondern auch ,ableitbare syntaktisch komplexe Kon-

4 Martine Dalmas (2009: 169) spricht bei Ausdrücken mit „metadiskursiver“ oder „metapragmatischer Funktion, unter denen sie auch „feste Phrasen wie damit meine ich oder ich meine oder ich glaube“ nennt von „Pseudo-Konnektoren“. Anders als „Konnektoren im engeren Sinne“, nämlich Konjunktionen und Satzadverbien mit anaphorischer Komponente, fungierten Pseudo- 
nektoren“ bzw. „frei bildbare Konnektoren“ mit aufgenommen, sofern sie als Kollokationen vergleichsweise usuell sind. Solche Einheiten müssen, wie HDK1 (ebd.) anmerkt, „nicht im Wörterbuch aufgeführt werden“. Die genannten Ausdrücke sind eindeutig von dieser Art. „Lexikalisierung“ im Sinne von nicht kompositional erschließbarer Verfestigung liegt in der Regel nicht vor - am ehesten noch bei gelinde gesagt oder der Verbindung genau genommen, die nicht auf einem Verbum dicendi beruht. Ich plädiere daher hier noch weitergehend dafür, von einem genuin syntaktischen Konstruktionsmuster auszugehen, das im Deutschen besonders stark und reihenbildend vertreten ist, während im Englischen oder Französischen neben gelegentlichen partizipialen (wie FRA autrement dit) überwiegend nicht partizipiale Konstruktionen in dieser Funktion eingesetzt werden, etwa Infinitivkonstruktionen wie ENG to tell the truth, FRA à vrai dire. Man beachte, dass bei den partizipialen Konstruktionen keine „Analepse“ im Sinne der IDS-Grammatik vorliegt: Die Konstruktion beruht als eigenständiger Konstruktionstyp nicht auf der Reduktion eines Satzes durch Weglassung von im Kontext gegebenen syntaktischen Einheiten. Rückverweisend im Text oder Diskurs werden die variablen Verbindungen wie (bereits/oben/zuvor, ...) gesagt/ausgeführt/erwähnt, ... gebraucht, während ein Vorverweis mithilfe der stärker verfestigten Verbindung wie folgt, also mithilfe einer Verbform 3SG Präsens Indikativ erfolgt. Hierzu vergleiche man auch die direkten Entsprechungen ENG as follows, FRA comme suit. Bei der prototypischen explizierenden Reformulierungsformel DEU das heißt (vgl. ENG that is, FRA c'est à dire) bleibt das syntaktische Konstruktionspotenzial des Verbs mit Subjekt und abhängigem Verbzweitsatz oder dassSatz-Komplement erhalten. Wenn allerdings nur eine Phrase, z.B. eine PP oder NP als Reformulierungsausdruck genannt wird, so ist syntaktische Identität mit dem Reformulandum gefordert; eine NP erscheint in der Regel im selben Kasus wie dieses. In diesem Fall also verhält sich die verbale Konstruktion wie ein Konjunktor:

(7) Als Held tritt der eingebildete Kranke, der Hypochondrist, der Geizige auf, das heißt ein Mensch, der andern und sich selber das Leben unnötig schwer macht. (Staiger, Emil: Grundbegriffe der Poetik: 197)

Konnektoren z.B. als Marker eines Perspektivenwechsels oder hätten modal abschwächende bzw. evidenzbetonende Funktion. In Dalmas (2001: 112f.) wird das heißt als Ausdruck von „Rephrasierung“ und/oder „Korrektur“ behandelt. 


\subsection{Umfunktionalisierung eines Phrasems zu einem propositionsbezogenen Modifikator}

Dieser, nach bisherigen Recherchen, eher seltenere Fall sei hier an ENG (besonders AmE) how come analysiert: Die Verbindung wird in Huddleston/Pullum (2002: 908) als „idiom“; bei Quirk et al. (1985: 840) als „stereotyped“ eingeordnet. Man vergleiche folgende Beispiele:

(8) How come the fridge is switched off? (Huddleston/Pullum 2002: 908)

(9) How come you know about it? / How come you speak such good Spanish? (BNC)

how come ist zwar funktional analog zu dem Frageadverb why, aber es induziert anders als why keine interrogative Syntax, also weder Inversion noch doUmschreibung. Vgl. im Kontrast:

(8a) Why is the fridge switched off?

(9a) Why do you speak so good Spanish?

Das heißt, syntaktisch verbindet sich how come mit der erfragten Proposition in Form eines kanonischen Aussagesatzes in SVO-Stellung, wobei how come die Linksversetzungsposition (left dislocation) einnimmt. Die Konstruktion ist damit noch rekonstruierbar als analog zur Spaltsatzkonstruktion (vgl. it-cleft-Konstruktion (Huddleston/Pullum 2002: 908)) wie in How is it you didn't tell me before?. Eine analoge Konstruktion dazu werden wir bei der periphrastischen Interrogativkonstruktion des Französischen weiter unten kennenlernen. Auch semantisch ist in vielen Fällen noch die Lesart 'Wie kommt es, dass ...' dominant. Vgl. Quirk et al (1985: 840) 'How does it come about (that) ...'.

Verwandt mit einem Adverb ist auch französisch voici/voilà. Die Ausdrücke gehen nach Grevisse/Goosse (2011: 1471) auf eine Form des Verbs voir + deiktischem Adverb ici (in der klitischen Form ci) bzw. là zurück. Man kann es als Effekt ihres verbalen Erbes betrachten, dass sie a) die Kookkurrenz mit einem finiten verbalen Prädikat nicht zulassen, sondern dieses in einer Aussage quasi ersetzen und b) wie das zugrundeliegende Verb Komplemente regieren kann (voici mes parents - les voilà).

Auch Verbindungen wie DEU koste es, was es wolle oder FRA coûte que coûte sind propositionsbezogen $\mathrm{zu}$ interpretieren, allerdings sind sie anders als etwa das prosodisch integrierte how come prosodisch desintegriert. Es handelt sich um emphatische Ausdrücke in der Bedeutung 'unbedingt, in jedem Fall, unter allen 
Umständen, um jeden Preis'. Es liegt eine Art 'Irrelevanzausdruck' vor, der Parallelen $\mathrm{zu}$ den weiter unten $\mathrm{zu}$ behandelnden Indefinitkonstruktionen aufweist: ${ }^{5}$ Konjunktiv/subjonctif der beteiligten Verben in der Konfiguration mit einem freien Relativsatz des von Haspelmath (1997) so benannten 'want/please'-Typs (so im Deutschen) bzw. einer tautologischen Formel (so im Französischen).

\subsection{Umfunktionalisierung eines Phrasems zum Modifikator einer Quantifikation}

Hier sind zwei koordinative Phraseme zu nennen, DEU sage und schreibe, ENG give or take. Typische Verwendungen illustrieren jeweils die folgenden Beispiele:

(10) So wie sie es bereits in Kauf nahmen, daß der Preis binnen eines Jahres um sage und schreibe zweitausend Dollar in die Höhe schoß. (Frankfurter Allgemeine, 5.11.2005)

(11) It cost $€ 200$, give or take. (Cambridge Dictionary, Online)

In beiden Fällen wird eine numerische Quantifikation modifiziert: Während sage und schreibe auf die erstaunliche oder auch empörende Höhe des numerischen Wertes verweist, drückt give or take eine Genauigkeitsabschwächung aus. Grammatisch verhalten sich bei Ausdrücke unterschiedlich: Während sage und schreibe in der Regel prosodisch und syntaktisch integriert ist und in (10) als attributive Erweiterung des Numerales zu verstehen ist, ist give or take syntaktisch desintegriert und wird der ersten Nennung des numerischen Werts als Nachtrag beigegeben. Allerdings zeigen beide Ausdrücke eine gewisse syntaktische Variabilität, die mit semantischer Unbestimmtheit einhergeht. So kann sage und schreibe auch als propositionsbezogenes Supplement auftreten, wie in (12), wo Quantifikation Teil der Verbbedeutung von 'verdreifachen' ist. Die Interpretation changiert ohnehin zwischen einer 'intensivierend-steigernden' im Sinne einer Intensitätspartikel und einer bewertenden im Sinne einer Modalpartikel.

5 Die Irrelevanz einer Bedingung wird durch so genannte 'Irrelevanzkonditionale' ausgedrückt, für die es verschiedene syntaktische Muster gibt, vgl. IDS-Grammatik (1997: 2319-2322); zur Weiterentwicklung zu Subjunktoren vgl. Leuschner (2005). 
Und gar nicht stolz auf «meinen» Kanton war ich, als die fremdenfeindliche SVP zu Anfang dieses Jahres bei den Grossratswahlen ihre Sitze sage und schreibe verdreifachen konnte. (St. Galler Tagblatt, 6.6.2000)

Bei dem englische Phrasem give or take kann die Valenz der beiden Verben sozusagen „reaktiviert“ werden, indem der Unschärfespielraum in Form einer NP wie in (13) spezifiziert wird:

(13) It'll be ready at 6:00, give or take a few minutes. (Cambridge Dictionary, Online)

Beide Paarformeln gehen auf rhetorisch gemeinte Aufforderungen oder auch Wunschbekundungen (dann im Deutschen als Formen des Konjunktiv Präsens) an den Adressaten zurück, dem Autor gedanklich bei der Durchführung bestimmter Operationen zu folgen. Man vergleiche auch die formelhafte Wendung Man höre und staune, die ich aufgrund des realisierten Subjekts und der notwendig desintegrierten Verwendung nicht als UVK einordne.

\subsection{Umfunktionalisierung zu einer periphrastischen Interrogativ-/Adverbialkonstruktion}

Beide Fälle sind Spezialformen von zwei allgemeineren miteinander als „Herausstellungsstrukturen“ (Altmann 2009: 13) verwandten syntaktischen Konstruktionstypen, nämlich dem Spaltsatz (cleft sentence) und dem Sperrsatz (pseudo-ceft sentence); vgl. zum Deutschen Altmann (2009), zu Englisch versus Deutsch Fischer (2009) und Gast/Wiechmann (2012), zum Französischen Lambrecht (1996). Beim Spaltsatz handelt es sich formal um ein Satzgefüge aus übergeordneter Struktur, bestehend aus Pronomen im Neutrum + Kopula + NP im Nominativ als „Cleft-Ausdruck“ (Altmann 2009), + Relativsatz. ${ }^{6}$ Funktional entspricht der Cleft-Ausdruck einer stark fokussierten Konstituente eines einfachen Satzes (statt des untergeordneten Relativsatzes). Der Konstruktionstyp ist im Englischen wie im Französischen im Vergleich zum Deutschen besonders frequent vertreten. Dabei spielt die in diesen beiden Sprachen weniger ausgeprägte lineare Variabilität des Satzbaus eine Rolle, die einer Fokussierung durch einfache Änderung der linearen Ordnung im Wege steht.

6 Welche syntaktische Funktion das Pronomen, der Cleft-Ausdruck und der Relativsatz haben, ist umstritten (vgl. Altmann 2009) und kann an dieser Stelle nicht diskutiert werden. 
In der für das Französische charakteristischen periphrastischen Interrogativkonstruktion erscheint in Ergänzungsfragesätzen anstelle des Cleft-Ausdrucks in der entsprechenden Deklarativsatzvariante das Fragewort in Spitzenstellung; vgl. (15) versus (14):

(14) C’est Marie que j’ai rencontrée hier.

(15) Qui est-ce que j'ai rencontrée hier?

Lehmann weist in seinem Skript zu „Grammatik“ im Abschnitt zum Spaltsatz (www.christianlehmann.eu/ling/lg_system/grammar/morph_syn/index.html, Stand: 23.11.2017) explizit darauf hin, dass in „pronominalen Interrogativsätzen“, wie er sie nennt, die Spaltsatzkonstruktion „grammatikalisiert“ sei. Das funktionale Merkmal von Spaltsätzen sei insofern als erfüllt zu betrachten, als „die erfragte Konstituente eines pronominalen Interrogativsatzes immer im Fokus“ sei. Ergänzend hinzuzusetzen ist, dass daneben eine Variante der Periphrase ohne Fragepronomen für den Entscheidungsfragesatz existiert, vgl. (16):

(16) Est-ce que tu as rencontré Marie hier?

Hier kann nicht mehr von einer Grammatikalisierung des Spaltsatzes im engeren Sinne die Rede sein. Es liegt jedoch auch hier Fokussierung vor, und zwar ein Verum-Fokus im Sinne von Höhle (1992).

Die englische periphrastische Adverbialkonstruktion ist eine Variante des Sperrsatzes, des pseudo-clefts. Sperrsätze bestehen aus einem w-Relativsatz im Deutschen bzw. wh-Relativsatz im Englischen gefolgt von Kopula + CleftAusdruck.

Betrachten wir folgenden Beleg für eine periphrastische Adverbialkonstruktion:

(17) And carbon dioxide is a major factor in global warming. That's why, on World Environment Day, a minimum of five million trees will be planted throughout Mexico. (BNC)

Die Umformung zu einem kanonischen Sperrsatz führt zu folgendem grammatisch randständigen und informationsstrukturell unangemessenen Ergebnis:

(17a) ??And carbon dioxide is a major factor in global warming. Why [...] a minimum of five million trees will be planted throughout Mexico, is that. 
Erst die Umstellung zu einem 'invertierten Sperrsatz' (Altmann 2009: 13) führt zu der mit (17) belegten wohlgeformten Struktur, in der das betonte Demonstrativum (als Cleft-Ausdruck) auf den mit dem vorangehenden Satz genannten Grund zurückverweist. In dieser halb-festen Form <Demonstrativum that + klitische Form 's des Kopulaverbs + durch ein Relativadverb eingeleiteter Satz $>$ ist die Konstruktion usuell; für that's why finden sich im Korpus BYU-BNC 2757 Belege, für that's where/how jeweils um 1000 und für that's when knapp 500 Belege. Die elaborierten, nicht verfestigten Formen, z.B. in Form von that's the reason why, that's the place where, that's the time when sind jeweils nur mit unter einem Dutzend Vorkommen im BNC belegt. Anders als bei verbargument- bzw. verbkomplementbezogenen Spaltsatzkonstruktionen (vgl. Beispiel (14)) ist der semantische Beitrag des Cleft-Ausdrucks hier direkt aus der lexikalischen Bedeutung des Relativadverbs (why, where, how, when) erschließbar, und zwar jeweils als vorerwähnter 'Grund', 'Ort', 'Art und Weise' oder 'Zeitpunkt' des Geschehens. Die explizite Benennung durch entsprechende NPs ist redundant. Die Konstruktionen entsprechen im Deutschen, wo die entsprechende invertierte SperrsatzKonstruktion zwar vereinzelt belegt, aber nicht üblich ist, somit anadeiktischen proadverbialen Verweisen, also DEU deshalb, dort, so und da/damals (mit Fokusakzent).

\subsection{Umfunktionalisierung zum Teil einer periphrastischen Indefinitkonstruktion}

In Haspelmath (1997) wird eine Reihe von verbalen Konstruktionstypen vorgestellt, die sprachübergreifend einer „Grammatikalisierung“, wie er es nennt, zu Indefinitpronomina, vorwiegend solche einer Zufallswahl, also im Sinne von 'irgendeiner, jeder beliebige' zugrunde liegen. Er nennt folgende Typen: den 'dunno'-Typ (bzw. 'I don't know'-Typ), den 'want/please'-Typ, den 'it may be'-Typ und den 'no matter'-Typ. Die deutschen und englischen Muster Gott/wer weiß + w-Wort / God/Heaven knows + wh-Wort sind UVK-Instanzen einer Variante des 'dunno'-Typs, während das französische Muster qu-Wort + que ce soit zum 'it may be'-Typ, 'n'importe + qu-Wort zum 'no-matter'-Typ gehört. Alle genannten sind nur schwach grammatikalisiert, während z.B. im Lateinischen mit den Indefinitpronomina quivis (> qui(s) 'wer' vis 'du willst') und quilibet (> qui(s) 'wer' libet 'es beliebt') univerbierte, stärker grammatikalisierte UVK des 'dunno'- und des 'want/please'-Typs vorliegen. 


\section{Tendenzen für mögliche Ausgangs- und Zielkonstruktionen bei UVK}

Wie deutlich geworden sein dürfte, ist das bisher ermittelte Material zu beschränkt und zu heterogen, um klare Aussagen über die möglichen Ausgangs- und Zielkonstruktionen und deren Korrelation bei UVK zu machen. Ich beschränke mich auf die Nennung einiger weniger erkennbarer Tendenzen.

- Als Verbklassen kommen neben dem Kopulaverb ‘sein' in erster Linie Verba dicendi und sentiendi vor.

- Die Verben bilden in unterschiedlichen Verbformen, jedoch bei Finitheit beschränkt auf Präsens Indikativ/Konjunktiv und 3. sowie 1. Person Singular, eine UVK.

- Bei den Ausgangskonstruktionen sind drei Typen zu nennen: Beschwörungsformeln bzw. andere emphatische Ausdrücke (= Typ 1), Konstruktionen, mit denen propositionale Einstellungen bzw. Modalisierungen bekundet werden (= Typ 2) sowie diskursorganisatorische oder metakommunikative Aussagen (= Typ 3).

- Die Typen 1 und 2 werden primär zu Modalpartikeln bzw. modalpartikelähnlichen Ausdrücken refunktionalisiert, während Typ 3 funktional einer Konnektivpartikel ähnelt.

- Verbindungen aus Demonstrativum und der Kopula werden im Englischen und Französischen als feste Bestandteile in periphrastischen Interrogativund Adverbialkonstuktionen genutzt. Es handelt sich dabei um Spezialisierungen von Spalt- und Sperrsatzkonstruktionen.

\section{Umfunktionalisierte verbale Konstruktionen als Instanzen von Grammatikalisierung und/oder Lexikalisierung?}

Heine/Kuteva (2007) nennen die Entwicklung der lateinischen Ausdrücke scilicet (> scire 'wissen' + licet 'es ist erlaubt') und videlicet (> videre 'sehen' + licet), also einer verkürzten und univerbierten verbalen Konstruktion aus einem Infinitiv und einem Finitum in der 3. Person Singular, zum „Adverb“ in der Bedeutung 'natürlich, selbstverständlich' als Beispiel für einen Grammatikalisierungsprozess. Sie betrachten Übertritte aus den Hauptwortarten Substantiv, Adjektiv und Verb in die kleineren Wortarten, etwa Präposition, Konjunktion oder Adverb, generell als Erscheinungsformen der Grammatikalisierung. Nach diesem Vorbild wären die 
Entwicklungen zumindest der univerbierten UVK, also etwa von maybe, peut-être, scheints, gottseidank, zu Modalpartikeln - in der neueren Grammatikschreibung werden ja solche Ausdrücke aus der traditionell umfassenden Klasse der Adverbien ausgegliedert - als Grammatikalisierungen zu verstehen. Dahinter verbirgt sich die Vorstellung, dass die Hauptwortarten als die eigentlichen Inhaltswortarten dem Lexikon zuzurechnen sind, die „Nebenwortarten“ eher der Grammatik. Lehmann (2002: 1) hält dieser Sehweise zu Recht entgegen, dass „minor word classes are not necessarily classes of grammatical formatives“. Es gebe etwa unter den Präpositionen „lexikalischere“ und „grammatischere“ Elemente; den lexikalischen Adverbien (etwa spanisch atrás 'hinten') stünden die eher grammatischen deiktischen Adverbien (wie spanisch ahí 'dort') gegenüber. Somit sei etwa die Entstehung einer Präposition aus einem relationalen Substantiv (wie bei spanisch $a$ base (de), deutsch auf Grund (von)) zuallererst ein Prozess der Bereicherung des lexikalischen Inventars, also der 'Lexikalisierung'. Entsprechend wäre also die Entstehung der oben genannten neuen Modalpartikeln Lexikalisierung. Auch nicht-univerbierte Phraseme, wie how come oder sage und schreibe müssen als Ganzes gelernt und im mentalen Lexikon gespeichert werden, sie wären also Fälle einer Mehr-Wort-Lexikalisierung, für die die prototypische Korrelation von strukturellem Wortstatus und Status als lexikalischer Einheit nicht gilt. ${ }^{7}$ Insofern als die aus verbalen Konstruktionen hervorgehenden lexikalischen Einheiten, die wir betrachtet haben, etwa neben how come auch peut-être oder Weiß Gott, einen klaren lexikalischen Gehalt haben und eher der „lexikalischeren“ als der ,grammatischeren“ Subklasse ihrer Wortklasse angehören, liegt hier keine Grammatikalisierung vor. Allerdings könnte sich an die Lexikalisierung auch ein Prozess zunehmender Grammatikalisierung anschließen, etwa wenn DEU scheints zu einem reinen Evidentialitätsmarker ausgebleicht würde. Nur die UVK, die als Teile von periphrastischen Konstruktionen analysiert wurden, fallen so gesehen in die Domäne der Grammatikalisierung. Bei ihnen spricht auch der Gebrauch des Kopulaverbs, also eines Verbs ohne spezifischen semantischen Gehalt, sowie Interaktion mit den sprachspezifischen grammatischen Hervorhebungsverfahren, für Grammatikalisierung.

Was die klassischen von Lehmann (2015: 129-188) herausgearbeiteten Parameter der Grammatikalisierung angeht, die insgesamt einen Verlust an Autonomie auf der paradigmatischen und syntagmatischen Achse beinhalten, so können diese auch auf die Bestandteile einer UVK bzw. allgemeiner einer Lexi-

7 Die in Abschnitt 3.2 behandelten freien oder halb-festen metakommunikativen Verbindungen (ehrlich/kurz/gelinde gesagt usw.) zeigen, dass es einen graduellen Übergang von rein „konstruktionalen“ zu lexikalisierten Umfunktionalisierungen gibt. 
kalisierung, zutreffen. Dies ist, nach Lehmann (2002: 15), auf ihre Gemeinsamkeit als reduktive Prozesse zurückzuführen, durch die die Freiheit des Sprechers bei der Wahl und Kombination der Bestandteile eines komplexen Ausdrucks eingeschränkt werde. ${ }^{8}$ Wenn wir somit die im Vorangehenden geschilderten Formen der Umfunktionalisierung verbaler Konstruktionen mit Ausnahme der Periphrasen (vgl. Abschnitte 3.5, 3.6) am ehesten als Lexikalisierungen einstufen, geht es hier allerdings nicht wie sonst unter dem Stichwort Lexikalisierung üblich primär um idiosynkratische Prozesse, die nur das einzelne verbale Syntagma betreffen, sondern um Tendenzen auf Kategorienebene, die verbalen Subklassen bzw. der Verbkategorie insgesamt, ihrer Syntax und Semantik, geschuldet sind. Insofern gibt es hier einen weiteren Berührungspunkt zwischen (so verstandener) Lexikalisierung und Grammatikalisierung: Verbale Konstruktionen erweisen sich kraft ihres syntaktischen und semantischen Potenzials als besonders geeignet, sekundär Funktionen im grammatischen Reich der Modalisierung, Abstufung und Kommentierung zu übernehmen. Dies ist ein weites Feld, das es zu bearbeiten gilt.

\section{Literatur}

Altmann, Hans (2009): Cleft- und Pseudocleft-Sätze (Spalt- und Sperrsätze) im Deutschen. (= Deutsche Sprachwissenschaft international 3). In: Brdar-Szabó, Rita/Knipf-Komlósi, Elisabeth/Péteri, Attila (Hgg.): An der Grenze zwischen Grammatik und Pragmatik. Frankfurt a.M.: Lang. 11-34.

Auer, Peter/Günthner, Susanne (2005): Die Entstehung von Diskursmarkern im Deutschen - ein Fall von Grammatikalisierung? In: Leuschner, Torsten/Mortelmans, Tanja/De Groodt,

8 Auer/Günthner (2005) diskutieren, ob die Entstehung von Diskursmarkern ebenfalls als Form von Grammatikalisierung betrachtet werden kann. Die Fälle, die sie behandeln, zeigen mit dem Gebrauch von verbalen Konstruktionsmustern mit Verba dicendi/sentiendi in der 1. oder 2. Person Singular wie in ich mein/glaub/weiß ich/woisch (schwäbisch für weißt du) und dem Imperativ wie in komm, sag mal Überschneidungen mit den hier erörterten UVK auf. Bemerkenswert sind insbesondere auch Fälle, bei denen Univerbierung und phonetische Reduktionen vorliegen, wie etwa bei dem genannten woisch oder bei coudon, das nach Dostie (2013: 16) im Quebecer Französisch für écoute donc gebraucht wird. Anders als bei den hier diskutierten Fällen ist mit dem Gebrauch als Diskursmarker allerdings weder ein propositionaler oder auch illokutionsbezogener Geltungsanspruch artikuliert noch ein metakommunikativer Kommentar explizit formuliert. Die Autoren sprechen hier zutreffend auch von einem gegenüber dem propositionalen, satzgrammatischen Bereich, erweiterten Skopus. Zum Verhältnis zwischen „modal particles“ (also Abtönungspartikeln im Sinne der IDS-Grammatik) und „discourse markers“ vgl. auch Degand/ Cornillie/Pietrandrea (Hgg.) (2013). 
Sarah (Hgg.): Grammatikalisierung im Deutschen. (= Linguistik - Impulse \&Tendenzen 9). Berlin/New York: De Gruyter. 335-362.

Dalmas, Martine (2001): Perspektivenwechsel durch Konnektoren und Formen mit konnektorenähnlicher Funktion. (= Eurogermanistik 16). In: Cambourian, Alain (Hg.): Textkonnektoren und andere textstrukturierende Einheiten. Tübingen: Stauffenburg. 109-127.

Dalmas, Martine (2009): Zur Bedeutungsbeschreibung von einigen Pseudo-Konnektoren. In: Henn-Memmesheimer, Beate (Hg.): Die Ordnung des Standards und die Differenzierung der Diskurse. Akten des 41. Linguistischen Kolloquiums. Frankfurt a.M: Lang. 167-178.

Degand, Liesbeth/Cornillie, Bert/Pietrandrea, Paola (Hgg.) (2013): Discourse markers and modal particles. Categorization and description. (= Pragmatics and Beyond New Series 234). Amsterdam/Philadelphia: Benjamins.

Diewald, Gabriele (2013): "Same same but different" - Modal particles, discourse markers and the art (and purpose) of categorization: In: Degand, Liesbeth/Cornillie, Bert/Pietrandrea, Paola (Hgg.). 19-45.

Dostie, Gaétane (2013): Les associations de marqueurs discursifs. De la cooccurrence libre à la collocation. Linguistik online 62, 5. 15-45. (www.linguistik-online.de/62_13/dostie.pdf, Stand: 23.11.2017).

Fischer, Klaus (2009): Cleft sentences. Form, function, and translation. In: Journal of Germanic Linguistics 21, 2. 167-191.

Gast, Volker/Wiechmann, Daniel (2012): W(h)-Clefts im Deutschen und Englischen: eine quantitative Untersuchung auf Grundlage des Europarl-Korpus. In: Gunkel, Lutz/Zifonun, Gisela (Hgg.): Deutsch im Sprachvergleich: Grammatische Kontraste und Konvergenzen. (= Jahrbuch des Instituts für Deutsche Sprache 2011). Berlin/Boston: De Gruyter. 333-362.

Ghezzi, Chiara/Molinelli, Piera (Hgg.) (2014): Discourse and pragmatic markers from Latin to the Romance languages. (= Oxford studies in diachronic and historical linguistics 9). Oxford, UK: Oxford University Press.

Gunkel, Lutz et al. (2017): Grammatik des Deutschen im europäischen Vergleich. Das Nominal. 2 Bde. (= Schriften des Instituts für Deutsche Sprache 14). Berlin/New York: De Gruyter.

Grevisse, Maurice/Goosse, André (2011): Le bon usage. Grammaire française. 15. Aufl. Brüssel: de Boeck/Duculot.

Haspelmath, Martin (1997): Indefinite pronouns. Oxford/New York: Clarendon Press/Oxford University Press.

HDK1 (2003) = Pasch, Renate et al. (2003): Handbuch der deutschen Konnektoren 1: Semantik der deutschen Satzverknüpfer. 2 Bde. (= Schriften des Instituts für Deutsche Sprache 9). Berlin/New York: De Gruyter.

HDK2 (2014) = Breindl, Eva/Volodina, Anna/Waßner, Ulrich Hermann (2014): Handbuch der deutschen Konnektoren 2: Semantik der deutschen Satzverknüpfer. 2 Bde. (= Schriften des Instituts für Deutsche Sprache 13). Berlin/Boston: De Gruyter.

Heine, Bernd/Kuteva, Tania (2007): The genesis of grammar. A reconstruction. (= Studies in the evolution of language 9). Oxford, UK: Oxford University Press.

Höhle, Tilman N. (1992): Über Verum-Fokus im Deutschen. In: Jacobs, Joachim (Hg.): Informationsstruktur und Grammatik. (= Linguistische Berichte. Sonderheft 4). Opladen: Westdeutscher Verlag. 112-141.

Huddleston, Rodney D./Pullum, Geoffrey K. (2002): The Cambridge grammar of the English language. Cambridge/New York: Cambridge University Press.

IDS-Grammatik (1997) = Zifonun, Gisela/Hoffmann, Ludger/Strecker, Bruno (1997): Grammatik der deutschen Sprache. 3 Bde. (= Schriften des Instituts für Deutsche Sprache 7). Berlin/ New York: De Gruyter. 
Lambrecht, Knud (1996): Information structure and sentence form: topic, focus, and the mental representations of discourse referents. (= Cambridge studies in linguistics 71). Cambridge: University Press.

Lehmann, Christian (1985): Grammaticalization: Synchronic variation and diachronic change. In: Lingua e Stile 20. 303-318.

Lehmann, Christian (2002): New reflections on grammaticalization and lexicalization. In: Wischer, Ilse/Diewald, Gabriele (Hgg.): New reflections on grammaticalization. Amsterdam/Philadelphia: Benjamins (TSL 49). 1-18.

Lehmann, Christian (2015): Thoughts on grammaticalization. 3. Aufl. (= Classics in Linguistics 1). Berlin: Language Science Press.

Leuschner, Torsten (2005): Ob blond, ob braun, ich liebe alle Frau'n. Irrelevanzkonditionalen als grammatikalisierter Diskurs. In: Leuschner, Torsten/Mortelmans, Tanja/De Groodt, Sarah (Hgg.): Grammatikalisierung im Deutschen. (= Linguistik - Impulse \&Tendenzen 9). Berlin/New York: De Gruyter. 279-307.

Meillet, Antoine (1948): L'évolution des formes grammaticales. In: Meillet, Antoine (1948): Linguistique historique et linguistique générale 1. (= Collection linguistique 8). Paris: Champion. 130-148. [Erstveröffentlichung 1912. In: Scientia (Rivista di Scienza) 12, 26. 6] Quirk, Randolph et al. (1985): A comprehensive grammar of the English language. London/ $\mathrm{New}$ York: Longman. 